\title{
Montreal cognitive assessment reflects cognitive reserve
}

\author{
Jae Myeong Kang ${ }^{1 \dagger}$, Young-Sung Cho ${ }^{2,3+}{ }^{\dagger}$, Soowon Park ${ }^{4}$, Byung Ho Lee ${ }^{5}$, Bo Kyung Sohn ${ }^{2}$, Chi Hyun Choi ${ }^{3}$, \\ Jeong-Seok Choi ${ }^{2,3}$, Hee Yeon Jeong ${ }^{2,3}$, Seong-Jin Cho', Jae-Hong Lee ${ }^{6}$ and Jun-Young Lee ${ }^{2,3^{*}}$ (D)
}

\begin{abstract}
Background: The Montreal Cognitive Assessment (MoCA) is known to have discriminative power for patients with Mild Cognitive Impairment (MCI). Recently Cognitive Reserve (CR) has been introduced as a factor that compensates cognitive decline. We aimed to assess whether the MoCA reflects CR. Furthermore, we assessed whether there were any differences in the efficacy between the MoCA and the Mini-Mental State Examination (MMSE) in reflecting CR.

Methods: MoCA, MMSE, and the Cognitive Reserve Index questionnaire (CRlq) were administered to 221 healthy participants. Normative data and associated factors of the MoCA were identified. Correlation and regression analyses of the MoCA, MMSE and CRlq scores were performed, and the MoCA score was compared with the MMSE score to evaluate the degree to which the MoCA reflected CR.

Results: The MoCA reflected total CRlq score (CRI; $B=0.076, P<0.001)$, CRI-Education $(B=0.066, P<0.001)$, and CRI-Working activity $(B=0.025, P=0.042)$, while MMSE reflected total CRI $(B=0.044, P<0.001)$ and CRIEducation ( $B=0.049, P<0.001)$ only. The MoCA differed from the MMSE in the reflection of total CRI $(Z=2.30)$.

Conclusion: In this study, we show that the MoCA score reflects CR more sensitively than the MMSE score. Therefore, we suggest that MoCA can be used to assess CR and early cognitive decline.
\end{abstract}

Keywords: MoCA, Cognition, Cognitive reserve, Dementia, Mild cognitive impairment

\section{Background}

In recent years, the number of patients with dementia has increased worldwide. This increase emphasizes the importance of early detection and treatment of dementia. Therefore, the development and standardization of effective screening tools are required. The Montreal Cognitive Assessment (MoCA) is known to distinguish patients with Mild Cognitive Impairment (MCI) from the normal population [1]. MoCA has shown higher sensitivity in detecting cognitive decline than the Mini-Mental State Examination (MMSE) [2], another common clinical screening tool for Alzheimer's disease (AD). Previous studies have indicated that the MoCA

\footnotetext{
* Correspondence: benji@snu.ac.kr

${ }^{\dagger} J a e$ Myeong Kang and Young-Sung Cho contributed equally to this work. ${ }^{2}$ Department of Psychiatry, SMG-SNU Boramae Medical Center, Boramae-Ro 5-Gil, Shindaebang-dong, Dongjak-gu, Seoul, Republic of Korea

${ }^{3}$ Department of Psychiatry and Behavioral Science, Seoul National University College of Medicine, Boramae-Ro 5-Gil, Shindaebang-dong, Dongjak-gu, Seoul, Republic of Korea

Full list of author information is available at the end of the article
}

exhibits high sensitivity and specificity in other languages as well. Moreover, MoCA is not only highly sensitive in identifying patients with $\mathrm{AD}$, but also non- $\mathrm{AD}$ patients who demonstrate behavioral variants of frontotemporal dementia [3], dementia associated with Parkinson's disease [4], and vascular dementia [5].

Cognitive Reserve (CR) is a concept based on the plasticity of the brain. CR is believed to counter the effects of aging or brain damage. It has been suggested that environmental factors play an important role in the onset of $\mathrm{AD}$. Moreover, a meta-analysis reported that higher CR lowers the risk for incidence of dementia to 54\% [6]. $\mathrm{CR}$ is associated with diverse factors of life experience such as higher intellectual quotient (IQ), education, occupational complexity and duration, and lifestyle [7]. Several measures have been developed to assess CR using these variables. The Cognitive Reserve Index questionnaire (CRIq), which has been developed by Nucci et al. [8], has advantages over other measures assessing comprehensive CR; CRIq measures 3 subdomains, i.e., 
education, occupation, and leisure activities, which are the most used proxies of CR [9]. Unlike other measurements, which only evaluate current activities of 1 or 2 domains, CRIq considers activities from all 3 subdomains throughout adulthood, including the frequency of the activities.

As the MoCA exhibits higher sensitivity than the MMSE in cognitive decline in the early stages of $A D$, the MoCA might be more sensitive to factors such as age, sex, and CR than MMSE. Recently, a study reported that educational domain in CR can affect MoCA and MMSE scores in patients with MCI [10]. However, to the best of our knowledge, no prior studies have assessed the degree to which MoCA reflects CR. Moreover, comparisons of the level at which MoCA and MMSE reflect CR are lacking. In the present study, we examined the associations of demographic factors (sex, age, and education) and $\mathrm{CR}$ with MoCA and compared the level of reflection of $\mathrm{CR}$ in MoCA and MMSE using CRIq as a comprehensive measure of $C R$.

\section{Methods \\ Subjects}

Subjects were recruited from a community-based center from March 2013 to June 2016 through recruitment announcements. Assessments of their physical and neuropsychiatric disorders were performed by 2 community dementia center consultant psychiatrists with 6 years and 15 years of experience, respectively.

Subjects with dementia or any mental or physical disease that may affect cognitive functioning, such as alcohol or other substance abuse, history of infarction, any evidence of central nervous system disorders or brain damage were excluded. Patients with severe major depressive disorders, altered state of consciousness like delirium, severe loss of hearing or sight, or language disorders were also excluded. However, patients with general medical problems, well-controlled diabetes, essential hypertension, or mild impairment of vision or hearing due to aging were not excluded if the impairment did not restrict their ability to perform the tests. Exclusion criteria were determined by psychiatrists on the basis of diagnostic criteria in the Diagnostic and Statistical Manual of Mental Disorders, fourth Edition (DSM-IV) [11].

A total of 221 subjects participated in this study and the age of subjects ranged from 60 to 90 years. Informed consent was obtained from all participants and the study was approved by the institutional review board of SMG-SNU Boramae Medical Center.

\section{Data collection}

Clinical neuropsychological tests (MoCA and MMSE) were administered to all subjects by mental health center specialists and professional dementia researchers (nurses, clinical psychologists) who had experience with the tests for an average of 10 years. CR was assessed using the self-reported CRIq completed by the subjects and interviews with the subjects' close family members.

\section{Measurements}

Korean version of Montreal cognitive assessment (MoCA-K) $\mathrm{MoCA}$ is a screening instrument to detect MCI developed by Nasreddine et al. Administration of MoCA takes about 10 to $15 \mathrm{~min}$. Higher scores indicate better cognition; the maximum score is 30 [2]. There are 12 items for cognitive domains; memory is tested by a short-term memory recall task (5 points); visuospatial ability is tested using a clock-drawing test (CDT; 3 points) and a 3-dimensional cube copy (1 point); executive function is tested using a trail-making test, part B (TMT-B; 1 point), a phonemic fluency task (1 point), and a 2 -item verbal abstraction task ( 2 points); attention, concentration, and working memory is tested using a sustained attention task (1 point), a serial subtraction task (3 points), and digits forward and backward tasks (1 point each); language is tested using a 3-item confrontation naming task with low-familiarity animals (lion, camel, rhinoceros; 3 points) and repetition of 2 syntactically complex sentences ( 2 points); orientation in time and place was also tested (6 points). MoCA-K was standardized for Koreans. It should be taken into consideration that the words for the short-term memory recall task and the TMT-B were replaced by Korean words and the semantic fluency task was replaced by a phonemic fluency task. One point was added for subjects with 6 years or less of education in MoCA-K to account for the large illiteracy in elderly Koreans [12]. In the current study, however, we did not add the correction point, to investigate normative data of MoCA-K without any adjustment.

\section{Mini-mental state examination-dementia screening (MMSE-DS)}

MMSE is the most commonly used dementia screening tool that can be performed in the relatively short time of 5 to $10 \mathrm{~min}$. The MMSE consists of 30 questions with a maximum score of 30 . Higher scores indicate better cognition. The MMSE tests the following 7 cognitive domains: orientation in time and place, memory registration and recall, attention and calculation, and language. In Korea, there are several standardized forms of the MMSE, such as MMSE-K [13], K-MMSE [14], MMSE$\mathrm{KC}$ [15]. However, the present study was conducted using MMSE-DS [16]. MMSE-DS has been developed to reflect the specific characteristics of the Korean elderly population and add cultural sensitivity. Normative data and test accuracy were validated for the Korean elderly population using age, sex, and years of education. Scores under 25 indicate cognitive impairment [16]. 
Korean version of cognitive reserve index questionnaire (K-CRIq) The CRIq has been developed by Nucci et al. It consists of 20 questions collecting demographic information, the number of years of education, and occupational and leisure activities throughout adulthood [8]. Regarding the years of education (CRI-Education), both formal and non-formal education and training years were included. The working activity (CRI-WorkingActivity) value is divided into 5 levels depending on the cognitive load involved. The leisure activity area (CRI-LeisureTime) is measured by evaluating cognitive activity, except for education and occupation activity, using 17 questions to evaluate the type and frequency of cognitive activity. Considering the effect of aging, scores for each category are obtained using age as an independent variable. The scores of 3 domains are calculated again to an average of 100 and a standard deviation of 15 to obtain the total CRIq score (CRI). Choi et al. reported a Korean version of CRIq normalized to age and sex [17].

\section{Statistical analyses}

Descriptive statistics were used to obtain the mean score and standard deviation for demographic characteristics and scores for MMSE, MoCA, and CRIq. Independent $t$-tests were used to compare the scores of different groups divided by sex. To measure correlations between the demographic variables and MoCA scores, Pearson correlation, and multivariate linear regression analyses (independent variable: age, sex, and years of education) were used. The interactions between age and years of education in MoCA were analyzed by multivariate regression analysis using interaction terms.

Univariate and multivariate linear regression analyses, using a stepwise method, were used to evaluate the correlation between CR and the MoCA and MMSE scores. To avoid multicollinearity, the total CRI score was analyzed separately from subdomains of CRIq. In order to compare the degree to which the MoCA and MMSE scores reflect $\mathrm{CR}$, correlation coefficients were compared using Fisher's $r$-to- $z$ transformation, and regression coefficients were compared using $z$ transformation. All statistical analyses were performed using Statistical Package for the Social Sciences (SPSS) version 23.0 (SPSS, Inc., Chicago IL) and statistical significance was defined as $P<0.05$ (2-tailed).

\section{Results}

\section{Demographics and clinical characteristics}

Table 1 shows the demographic and clinical results of the subjects that participated in this study. CRIq is presented as the total score (CRI) and the 3 subdomains, i.e., CRI-Education, CRI-WorkingActivity, and CRI-LeisureTime. When the results of the male and female subjects were compared, male subjects showed significantly higher scores of
CRI, CRI-Education, and CRI-WorkingActivity $(P<0.001)$ than female subjects; female subjects exhibited a significantly higher CRI-LeisureTime score than male subjects $(P<0.001)$.

\section{Relationships between demographical variables and MoCA}

Table 2 shows descriptive data of MoCA scores using age, sex, and educational level. MoCA scores were associated with all 3 demographic variables, showing higher scores for subjects with longer educational times (correlation analysis: $r=0.446, P<0.001$ ), younger age (correlation analysis: $r=-0.347, P<0.001$ ), and male subjects (independent $t$-test: $t=2.903, P=0.004$ ).

When the demographic variables were analyzed using multivariate linear regression analysis, education level showed moderating effect on the influence of age on MoCA score. The higher the education level was, the lower the degree of MoCA score decreased with age ( $B=0.017, P=0.023$, Additional file 1: Table A1).

\section{Correlations of MoCA, MMSE, and CR}

Pearson correlation was performed between MoCA, MMSE and CRIq scores (Table 3).

Correlation of MoCA score with total CRI was $r=$ $0.383, \quad P<0.001$; with CRI-Education $r=0.356, \quad P$ $<0.001$; CRI-WorkingActivity $r=0.246, P<0.001$, and CRI-LeisureTime $r=0.224, P=0.001)$. Semi-partial correlation analysis adjusting sex was performed in order to find relation between MoCA, MMSE, and CRIq scores adjusting the effect of sex because age and years of education were already adjusted in CRIq and it showed comparable results (Additional file 1: Table A2).

Linear regression analyses were performed to investigate the effect of CRIq on the MoCA and MMSE scores (Table 4).

We conducted univariate linear regression analyses for total and each subdomain score of CRIq, and multivariate linear regression analyses using a stepwise method based on the 3 subdomains of CRIq. Total CRI and the 3 subdomains of CRIq were analyzed separately to avoid multicollinearity. The results showed a significant association of the total CRI and MoCA scores $(B=0.076$, $P<0.001)$, with an explanatory power of $15 \%\left(R^{2}=\right.$ 0.147, $F=37.723, P<0.001)$. In the final model of multivariate regression analysis using stepwise method $(F=18.245, \quad P<0.001)$, CRI-Education and CRI-WorkingActivity also showed correlations with the MoCA score (CRI-Education: $B=0.066, \quad P<0.001$; CRI-WorkingActivity: $B=0.025, P=0.042$ ), with an explanatory power of $14 \%\left(R^{2}=0.143\right)$. CRI-LeisureTime was not included in the final regression model. For the MMSE score, univariate linear regression analysis $(F=$ 35.416, $P<0.001)$ indicated that the total CRI was significantly correlated with the MMSE score $(B=0.044, P=0.042)$ 
Table 1 Demographic and clinical characteristics

\begin{tabular}{llll}
\hline & Male $(n=95)$ & Female $(n=126)$ & Total $(n=221)$ \\
\hline Age (year) & $74.60 \pm 5.54$ & $73.39 \pm 5.79$ & $73.91 \pm 5.70$ \\
$60-74$ & $50(52.6 \%)$ & $74(58.7 \%)$ & $124(56.1 \%)$ \\
$75-90$ & $45(47.4 \%)$ & $52(41.3 \%)$ & $97(43.9 \%)$ \\
Education (year) & $10.65 \pm 4.61$ & $8.83 \pm 4.81$ & $9.61 \pm 4.80$ \\
0-6 & $29(30.5 \%)$ & $56(44.4 \%)$ & $85(38.5 \%)$ \\
$7-12$ & $35(36.8 \%)$ & $38(30.2 \%)$ & $73(33.0 \%)$ \\
13- & $31(32.6 \%)$ & $32(25.4 \%)$ & $63(28.5 \%)$ \\
MMSE & $27.47 \pm 1.83$ & $26.94 \pm 2.23$ & $27.17 \pm 2.08$ \\
MoCA & $23.40 \pm 3.21$ & $22.03 \pm 3.65$ & $22.62 \pm 3.53$ \\
CRlq & & & $102.74 \pm 17.57$ \\
CRI & $107.32 \pm 18.46$ & $99.28 \pm 16.10$ & $102.23 \pm 16.19$ \\
CRI-Education & $106.11 \pm 15.63$ & $99.30 \pm 16.04$ & $101.86 \pm 19.49$ \\
CRI-WorkingActivity & $114.50 \pm 20.70$ & $92.32 \pm 11.55$ & $101.92 \pm 18.80$ \\
CRI-LeisureTime & $95.44 \pm 16.32$ & $106.80 \pm 19.13$ & 0.041 \\
\hline
\end{tabular}

Data are shown in mean \pm standard deviation or number (\%)

MMSE Mini-mental Status Examination, MoCA Montreal Cognitive Assessment, CRlq Cognitive Reserve Index questionnaire

and the explanatory power was $14 \%\left(R^{2}=0.139\right)$. When all 3 domains of CRIq were treated as independent variables in the multivariate linear regression analysis $(F=36.655$, $P<0.001)$, only CRI-Education was included in the final model with significant effects $(B=0.049, P<0.001)$. The explanatory power was $14 \%\left(R^{2}=0.143\right)$.

Table 2 Mean, standard deviation, and selected percentiles of the MoCA-K by age, educational level, and sex in the normal Korean elderly

\begin{tabular}{|c|c|c|c|c|c|c|}
\hline \multirow[t]{2}{*}{ Educational level (year) } & \multicolumn{3}{|c|}{ Male $(n=95)$} & \multicolumn{3}{|c|}{ Female $(n=126)$} \\
\hline & $0-6$ & $7-12$ & $13-$ & $0-6$ & $7-12$ & 13- \\
\hline \multicolumn{7}{|l|}{ Age (year) } \\
\hline \multicolumn{7}{|l|}{$60 \sim 74$} \\
\hline $\mathrm{N}$ & 16 & 19 & 15 & 33 & 24 & 17 \\
\hline Mean & 23.44 & 24.05 & 25.33 & 21.88 & 23.54 & 25.12 \\
\hline Standard deviation & 2.80 & 3.47 & 1.92 & 3.55 & 2.89 & 2.37 \\
\hline Lower quartile & 21.25 & 22.0 & 24.0 & 19.0 & 21.0 & 23.0 \\
\hline Median & 23.0 & 25.0 & 26.0 & 23.0 & 23.50 & 26.0 \\
\hline Upper quartile & 25.50 & 27.0 & 27.0 & 24.50 & 26.0 & 26.0 \\
\hline \multicolumn{7}{|l|}{$75 \sim 90$} \\
\hline N & 13 & 16 & 16 & 23 & 14 & 15 \\
\hline Mean & 19.69 & 22.50 & 24.69 & 18.87 & 19.71 & 23.47 \\
\hline Standard deviation & 2.29 & 3.25 & 2.27 & 3.08 & 2.76 & 2.95 \\
\hline Lower quartile & 18.50 & 19.25 & 23.0 & 15.0 & 18.0 & 21.0 \\
\hline Median & 19.0 & 23.0 & 25.0 & 20.0 & 19.0 & 24.0 \\
\hline Upper quartile & 21.0 & 24.75 & 26.0 & 21.0 & 22.0 & 26.0 \\
\hline
\end{tabular}

MoCA-K Montreal Cognitive Assessment

\section{Comparison between MoCA and MMSE on reflection of CRIq}

Correlations of MoCA and MMSE scores with CRIq are shown in a scatter plot (Fig. 1). The zero-order correlation coefficients shown in Table 3 were compared using Fisher's $r$-to- $z$ transformation. We observed no significant difference in total CRI ( $r$ for MoCA $=0.383, r$ for MMSE $=0.373)$ and CRI-Education $(r$ for MoCA $=0.356$, $r$ for MMSE $=0.379$ ). In addition, we compared the multivariate regression coefficient $B$ shown in Table 4, which represents the correlation slope between the CRIq score and MoCA or MMSE scores; MoCA scores tended to show a larger slope than MMSE scores. We observed a significant difference in total CRI $(B$ for MoCA $=$ $0.076, B$ for $\mathrm{MMSE}=0.044, Z=2.30$ ) but not in CRI-Education $(B$ for $\mathrm{MoCA}=0.066, B$ for $\mathrm{MMSE}=$ $0.049, Z=1.06)$.

\section{Discussion}

In this study, the MoCA was associated with years of education, age, and sex. In addition, MoCA was also significantly associated with CR. Furthermore, we compared

Table 3 Correlation analyses between CRlq score and MMSE or MoCA scores

\begin{tabular}{lllll}
\hline & CRI & CRI-Education & CRI-WorkingActivity & CRI-LeisureTime \\
\hline MoCA & $r=0.383$ & $r=0.356$ & $r=0.246$ & $r=0.224$ \\
& $P<0.001$ & $P<0.001$ & $P<0.001$ & $P=0.001$ \\
MMSE & $r=0.373$ & $r=0.379$ & $r=0.165$ & $r=0.268$ \\
& $P<0.001$ & $P<0.001$ & $P=0.014$ & $P<0.001$ \\
\hline
\end{tabular}

CRIq Cognitive Reserve Index questionnaire, MMSE Mini-mental State Examination, MoCA Montreal Cognitive Assessment 
Table 4 Univariate and multivariate regression analyses between CRlq score and MoCA and MMSE scores

\begin{tabular}{|c|c|c|c|c|c|}
\hline Dependent variable & Independent variable & $B$ & Standard Error & $t$ & $P$ value \\
\hline \multicolumn{6}{|c|}{ Univariate regression analyses } \\
\hline \multirow[t]{4}{*}{ MoCA } & CRI & 0.076 & 0.012 & 6.14 & $<0.001$ \\
\hline & CRI-Education & 0.077 & 0.014 & 5.64 & $<0.001$ \\
\hline & CRI-WorkingActivity & 0.044 & 0.012 & 3.76 & $<0.001$ \\
\hline & CRI-LeisureTime & 0.042 & 0.012 & 3.39 & 0.001 \\
\hline \multirow[t]{4}{*}{ MMSE } & CRI & 0.044 & 0.007 & 5.95 & $<0.001$ \\
\hline & CRI-Education & 0.049 & 0.008 & 6.05 & $<0.001$ \\
\hline & CRI-WorkingActivity & 0.018 & 0.007 & 0.01 & 0.014 \\
\hline & CRI-LeisureTime & 0.030 & 0.007 & 4.11 & $<0.001$ \\
\hline \multicolumn{6}{|c|}{ Multivariate regression analyses ${ }^{a}$} \\
\hline \multirow[t]{2}{*}{ MoCA } & CRI-Education & 0.066 & 0.014 & 4.59 & $<0.001$ \\
\hline & CRI-WorkingActivity & 0.025 & 0.012 & 2.05 & 0.042 \\
\hline MMSE & CRI-Education & 0.049 & 0.008 & 6.05 & $<0.001$ \\
\hline
\end{tabular}

${ }^{a}$ Multivariate linear regression: independent variables are CRI-Education, CRI-WorkingActivity, and CRI-LeisureTime. The variables included in the final models of multivariate regression analyses using stepwise method are presented

CRIq Cognitive Reserve Index questionnaire, MoCA Montreal Cognitive Assessment, MMSE Mini-mental State Examination

the degree to which $\mathrm{CR}$ was reflected in the MoCA and MMSE. Our results showed that the MoCA score reflected CR better than the MMSE score.

The first findings of our study are the MoCA scores. MoCA scores tended to be higher for the patients with more years of education and of younger age. These results are similar to results from previous normative studies $[18,19]$. Additionally, aging had a larger effect on MoCA scores in a population with lower education than in a population with higher education. This result indicates
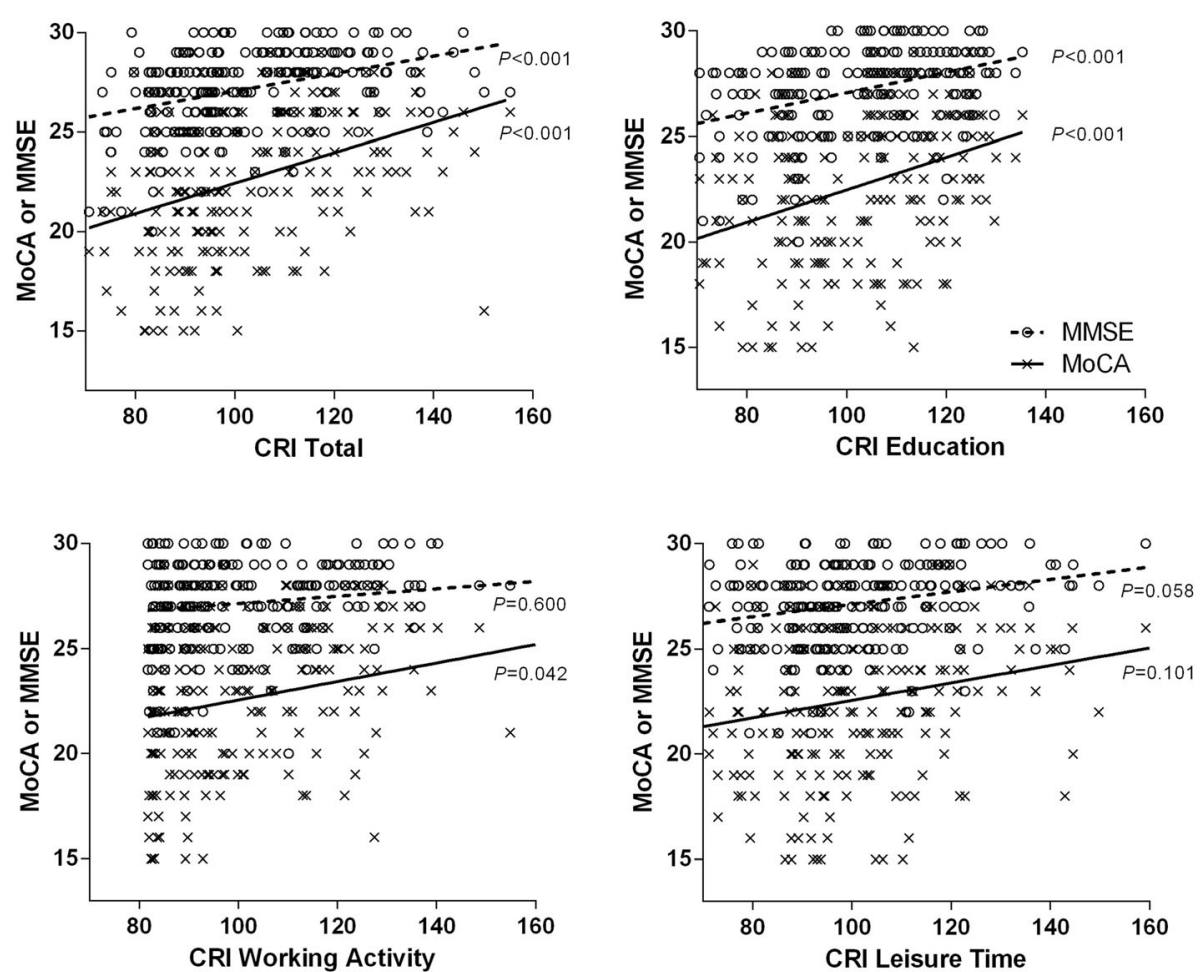

Fig. 1 Correlation between MoCA or MMSE and CRIq. $P$ values for the subdomains of CRlq are obtained from the multivariate regression analyses using stepwise method (dependent variable: MoCA or MMSE, independent variables: CRI-Education, CRI-WorkingActivity, CRILeisureTime). MoCA Montreal Cognitive Assessment, MMSE Mini-Mental State Examination, CRla Cognitive reserve index questionnaire 
that the effect of education overcomes the effect of aging. This is in line with previous studies suggesting that education is a major factor in CR [20,21]. Regarding this significant effect of education, Nasreddine et al. have included one correction point in the MoCA for individuals with education of 12 years and below. In Korea, Lee et al. set a similar correction point in the MoCA-K for individuals with 6 years or less of education, considering the low level of education in Korea [12]. However, in this study, the correction point was not applied to determine the association between normative scores of MoCA-K and CR or demographic variables. MoCA scores were higher in male subjects than in female subjects, which is in line with the results of the Chinese MoCA study [22] and an MMSE normative study performed on Koreans [23]. This sexual discrepancy in normative data is considered to reflect a tendency of elderly men to have more intellectual, social, and physical opportunities than women due to gender role differences.

Our results demonstrated that both $\mathrm{MoCA}$ and MMSE correlate with $\mathrm{CR}$, although MoCA score reflects CR more sensitively than MMSE score. In regression analyses with total and subdomain scores of CRIq, the MoCA score reflected total CRI, CRI-Education, and CRI-WorkingActivity, while the MMSE score only reflected total CRI and CRI-Education. In addition, the correlation slope between the total CRI and MoCA scores was significantly higher than that between the total CRI and MMSE scores. We suggest that this discrepancy was due to differences in the tools of assessment employed by the MoCA and MMSE; MoCA contains various assessment tools for frontal lobe function (TMT-B, copy of a cube, CDT, letter A tap, letter fluency), which are not included in MMSE, making it sensitive to and reflective of $C R$ in various cognitive subdomains [24]. It is known from previous studies that connectivity in the frontal lobe plays an important role in CR $[25,26]$. This can be more prominent in the elderly. Compared to young individuals, old individuals use different brain networks [27]. Scarmeas et al. investigated the brain regions related to $\mathrm{CR}$ and found that the inferior frontal region is related to CR only in old subjects [28]. The increase in activity and connectivity in the prefrontal area of patients with $\mathrm{AD}$, compared with normal controls, has been interpreted to reflect the recruitment of cognitive resources $[9,29]$. Increased activity in the prefrontal cortex has also been associated with tasks such as episodic, retrieval, and recognition memory, which are the most basic memory functions and are frequently affected by cognitive decline [25].

In particular, our study suggests that only the MoCA scores can sensitively reflect CRI-WorkingActivity among the subdomains of CRIq. This association between MoCA and the vocational ability is attributed to the assessment of frontal lobe function by MoCA. The effect of vocational ability on CR can be explained by the motivation to participate in cognitively stimulating daily activities, neuronal plasticity, and executive functions, making it a favorable domain to examine CR [30, 31]. Many studies have shown that vocational abilities are dependent on frontal lobe function in patients with traumatic brain injuries and vascular degenerative changes. Since the frontal lobe is involved in language, arithmetic processing, attention, planning and strategy application, and willful action, it can be a good indicator of vocational ability [32-34]. Proxies such as educational or occupational achievements, and IQ have been used to characterize CR previously [7, 31]. In conjunction with our results, it appears that it would be clinically beneficial to use MoCA as a brief cognitive screening tool for the assessment of both cognitive function and reserve.

Recently, interest in CR has increased because of its importance in identifying and managing patients with preclinical and prodromal AD. Lacking a current disease-modifying treatment, $C R$ can be another interesting candidate for the prevention and treatment of AD. Accordingly, the number of studies investigating treatments using cognitive stimulation is sharply increasing [35]. CR consists of these lifetime experiences. High levels of $\mathrm{CR}$ have been reported to be capable of lowering the risk of incidents of dementia, its clinical symptoms, and its pathologic changes, as shown by neuroimaging studies $[6,10,36-38]$.

Our study shows that MoCA score correlates with CR, especially in terms of education and working activity, which corresponds to executive function. Therefore, MoCA can be a useful tool to evaluate CR and to screen the subtle changes in cognition. However, our present study has limitations that should be taken into account when evaluating the results. For example, our study only included a small number of participants, and the participants in this study had fewer years of education than those in studies from other countries. Future larger studies supported by more validating methods and biological assays are required to overcome these limitations.

\section{Conclusion}

This study confirms that MoCA reflects CR, and that $\mathrm{CR}$ is reflected more sensitively by the MoCA score than the MMSE score. The clinical use of the MoCA is expected to increase markedly, because it provides an easy way to evaluate cognitive function and CR, without any additional tests or large-scale batteries. This study may provide valuable insight for future, large community-based studies of early cognitive decline and CR. 


\section{Additional file}

Additional file 1: Table A1. Moderating effect of Education on the relationship between Age and MoCA score. Table A2. Semi-partial correlation between CRlq and MMSE or MoCA scores adjusting sex. (DOCX $19 \mathrm{~kb}$ )

\section{Abbreviations}

CDT: Clock-Drawing Test; CR: Cognitive Reserve; CRlq: Cognitive Reserve Index questionnaire; DSM-IV: Diagnostic and Statistical Manual of Mental Disorders, fourth Edition; IQ: Intellectual Quotient; MCl: Mild Cognitive Impairment; MMSE: Mini-Mental State Examination; MoCA: Montreal Cognitive Assessment; TMT-B: Trail-Making Test, Part B

\section{Acknowledgements}

Not applicable.

\section{Funding}

This study was conducted without financial support. All authors are independent from the funding sources.

\section{Availability of data and materials}

The datasets analyzed during the current study are not publicly available, as consent for public disclosure was not obtained. Data are, however, available from the authors upon reasonable request and with permission of institutional review board of SMG-SNU Boramae Medical Center.

\section{Author's contributions}

All authors listed above contributed significantly to this study. JYL: study concept and design, critical review and approval for final manuscript; JMK, YSC: data acquisition, data analyses, writing and editing manuscript; SP: data analyses and interpretation; BHL: data analyses, critical review, and editing manuscript; JHL, BKS, JSC, HYJ, SJC: critical review; CHC: data acquisition, critical review, and editing manuscript. All authors have read and approved the final version of the manuscript.

\section{Ethics approval and consent to participate}

This study has been conducted according to the principles expressed in the Declaration of Helsinki. Written informed consent was obtained from all participants, and this study was approved by the institutional review board of SMG-SNU Boramae Medical Center.

\section{Consent for publication}

Not applicable.

\section{Competing interests}

The authors declare that they have no competing interests.

\section{Publisher's Note}

Springer Nature remains neutral with regard to jurisdictional claims in published maps and institutional affiliations.

\section{Author details}

${ }^{1}$ Department of Psychiatry, Gil Medical Center, Gachon University College of Medicine, Incheon, Republic of Korea. ${ }^{2}$ Department of Psychiatry, SMG-SNU Boramae Medical Center, Boramae-Ro 5-Gil, Shindaebang-dong, Dongjak-gu, Seoul, Republic of Korea. ${ }^{3}$ Department of Psychiatry and Behavioral Science, Seoul National University College of Medicine, Boramae-Ro 5-Gil, Shindaebang-dong, Dongjak-gu, Seoul, Republic of Korea. ${ }^{4}$ Department of Education, Sejong University, Seoul, Republic of Korea. ${ }^{5}$ Department of Psychology, Salisbury University, Salisbury, Maryland, USA. ${ }^{6}$ Department of Neurology, Asan Medical Center, University of Ulsan College of Medicine, Seoul, Republic of Korea.
Received: 15 June 2017 Accepted: 17 October 2018

Published online: 30 October 2018

\section{References}

1. Petersen RC, Smith GE, Waring SC, Ivnik RJ, Tangalos EG, Kokmen E. Mild cognitive impairment: clinical characterization and outcome. Arch Neurol. 1999;56(3):303-8.

2. Nasreddine ZS, Phillips NA, Bédirian V, Charbonneau S, Whitehead V, Collin I, Cummings JL, Chertkow $\mathrm{H}$. The Montreal cognitive assessment, MoCA: a brief screening tool for mild cognitive impairment. J Am Geriatr Soc. 2005:53(4):695-9.

3. Freitas S, Simões MR, Alves L, Duro D, Santana I. Montreal cognitive assessment (MoCA): validation study for frontotemporal dementia. J Geriatr Psychiatry Neurol. 2012;25(3):146-54.

4. Hoops S, Nazem S, Siderowf A, Duda J, Xie S, Stern M, Weintraub D. Validity of the MoCA and MMSE in the detection of $\mathrm{MCl}$ and dementia in Parkinson disease. Neurology. 2009;73(21):1738-45.

5. Freitas S, Simoes MR, Alves L, Vicente M, Santana I. Montreal cognitive assessment (MoCA): validation study for vascular dementia. J Int Neuropsychol Soc. 2012:18(06):1031-40.

6. Valenzuela MJ, Sachdev P. Brain reserve and dementia: a systematic review. Psychol Med. 2006:36(04):441-54.

7. Stern Y. Cognitive reserve in ageing and Alzheimer's disease. Lancet Neurol. 2012;11(11):1006-12.

8. Nucci M, Mapelli D, Mondini S. Cognitive reserve index questionnaire (CRlq): a new instrument for measuring cognitive reserve. Aging Clin Exp Res. 2012; 24(3):218-26.

9. Horwitz B, McIntosh AR, Haxby JV, Furey M, Salerno JA, Schapiro MB, Rapoport SI, Grady CL. Network analysis of PET-mapped visual pathways in Alzheimer type dementia. Neuroreport. 1995;6(17):2287-92.

10. Liu Y, Cai Z-L, Xue S, Zhou X, Wu F. Proxies of cognitive reserve and their effects on neuropsychological performance in patients with mild cognitive impairment. J Clin Neurosci. 2013:20(4):548-53.

11. American Psychiatric Association: Diagnostic and statistical manual of mental disorders DSM-IV-TR fourth edition (text revision). 2000.

12. Lee J-Y, Lee DW, Cho S-J, Na DL, Jeon HJ, Kim S-K, Lee YR, Youn J-H, Kwon M, Lee J-H. Brief screening for mild cognitive impairment in elderly outpatient clinic: validation of the Korean version of the Montreal cognitive assessment. J Geriatr Psychiatry Neurol. 2008;21(2):104-10.

13. Park JH, Kwon YC. Modification of the mini-mental state examination for use in the elderly in a non-western society. Part 1. Development of korean version of mini-mental state examination. Int J Geriatr Psychiatry. 1990;5(6): $381-7$.

14. Kang Y, Na DL, Hahn S. A validity study on the Korean mini-mental state examination (K-MMSE) in dementia patients. J Korean Neurol Assoc. 1997; 15(2):300-8.

15. Lee JH, Lee KU, Lee DY, Kim KW, Jhoo JH, Kim JH, Lee KH, Kim SY, Han SH, Woo J. Development of the Korean version of the consortium to establish a registry for Alzheimer's disease assessment packet (CERAD-K) clinical and neuropsychological assessment batteries. J Gerontol B Psychol Sci Soc Sci. 2002;57(1):P47-53

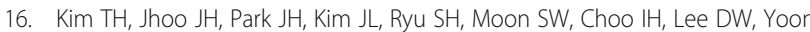
JC, Do YJ. Korean version of mini mental status examination for dementia screening and its' short form. Psychiatry investig. 2010;7(2):102-8.

17. Petersen RC, Roberts RO, Knopman DS, Boeve BF, Geda YE, Ivnik RJ, Smith GE, Jack CR. Mild cognitive impairment: ten years later. Arch Neurol. 2009; 66(12):1447-55.

18. Rossetti HC, Lacritz LH, Cullum CM, Weiner MF. Normative data for the Montreal cognitive assessment (MoCA) in a population-based sample. Neurology. 2011;77(13):1272-5.

19. Freitas S, Simões MR, Alves L, Santana I. Montreal cognitive assessment (MoCA): normative study for the Portuguese population. J Clin Exp Neuropsychol. 2011;33(9):989-96.

20. Roe CM, Xiong C, Miller JP, Morris JC. Education and Alzheimer disease without dementia support for the cognitive reserve hypothesis. Neurology. 2007:68(3):223-8.

21. Kemppainen NM, Aalto S, Karrasch M, Någren K, Savisto N, Oikonen V, Viitanen M, Parkkola R, Rinne JO. Cognitive reserve hypothesis: Pittsburgh compound $\mathrm{B}$ and fluorodeoxyglucose positron emission tomography in relation to education in mild Alzheimer's disease. Ann Neurol. 2008; 63(1):112-8 
22. Wen H, Zhang Z, Niu F, Li L. The application of Montreal cognitive assessment in urban Chinese residents of Beijing. Zhonghua Nei Ke Za Zhi. 2008;47(1):36-9.

23. Lee DY, Lee KU, Lee JH, Kim KW, Jhoo JH, Youn JC, Kim SY, Woo SI, Woo Jl. A normative study of the mini-mental state examination in the Korean elderly. J Korean Neuropsychiatr Assoc. 2002;41(3):508-25.

24. Julayanont P, Phillips N, Chertkow H, Nasreddine ZS. Montreal cognitive assessment (MoCA): concept and clinical review. In: Cognitive screening instruments. London: Springer; 2013. p. 111-51.

25. Grady CL, Mclntosh AR, Beig S, Keightley ML, Burian H, Black SE. Evidence from functional neuroimaging of a compensatory prefrontal network in Alzheimer's disease. J Neurosci. 2003;23(3):986-93.

26. Supekar K, Menon V, Rubin D, Musen M, Greicius MD. Network analysis of intrinsic functional brain connectivity in Alzheimer's disease. PLoS Comput Biol. 2008;4(6):e1000100.

27. Stern Y, Habeck C, Moeller J, Scarmeas N, Anderson KE, Hilton HJ, Flynn J, Sackeim $H$, van Heertum R. Brain networks associated with cognitive reserve in healthy young and old adults. Cereb Cortex. 2005;15(4):394-402.

28. Scarmeas N, Zarahn E, Anderson KE, Hilton J, Flynn J, Van Heertum RL, Sackeim HA, Stern Y. Cognitive reserve modulates functional brain responses during memory tasks: a PET study in healthy young and elderly subjects. Neuroimage. 2003;19(3):1215-27.

29. Jagust WJ, Friedland RP, Budinger TF, Koss E, Ober B. Longitudinal studies of regional cerebral metabolism in Alzheimer's disease. Neurology. 1988;38(6): 909-12.

30. Buchman AS, Bennett DA. Loss of motor function in preclinical Alzheimer's disease. Expert Rev Neurother. 2011;11(5):665-76.

31. Scarmeas N, Stern Y. Cognitive reserve: implications for diagnosis and prevention of Alzheimer's disease. Curr Neurol Neurosci Rep. 2004;4(5): 374-80.

32. Menon V, Rivera S, White C, Glover G, Reiss A. Dissociating prefrontal and parietal cortex activation during arithmetic processing. Neuroimage. 2000; 12(4):357-65.

33. Baldo JV, Shimamura AP, Delis DC, Kramer J, Kaplan E. Verbal and design fluency in patients with frontal lobe lesions. J Int Neuropsychol Soc. 2001; 7(05):586-96.

34. Frith CD, Friston K, Liddle PF, Frackowiak RS. Willed action and the prefrontal cortex in man: a study with PET. Proc Biol Sci. 1991;244(1311):241-6.

35. Winblad B, Palmer K, Kivipelto M, Jelic V, Fratiglioni L, Wahlund LO, Nordberg A, Bäckman L, Albert M, Almkvist O. Mild cognitive impairmentbeyond controversies, towards a consensus: report of the international working group on mild cognitive impairment. J Intern Med. 2004;256(3):240-6.

36. Scarmeas N, Zarahn E, Anderson KE, Habeck CG, Hilton J, Flynn J, Marder KS, Bell KL, Sackeim HA, Van Heertum RL. Association of life activities with cerebral blood flow in Alzheimer disease: implications for the cognitive reserve hypothesis. Arch Neurol. 2003;60(3):359-65.

37. Perneczky R, Drzezga A, Diehl-Schmid J, Schmid G, Wohlschläger A, Kars S, Grimmer T, Wagenpfeil S, Monsch A, Kurz A. Schooling mediates brain reserve in Alzheimer's disease: findings of fluoro-deoxy-glucose-positron emission tomography. J Neurol Neurosurg Psychiatry. 2006;77(9):1060-3.

38. Bennett DA, Wilson R, Schneider J, Evans D, De Leon CM, Arnold S, Barnes $\mathrm{L}$, Bienias J. Education modifies the relation of AD pathology to level of cognitive function in older persons. Neurology. 2003;60(12):1909-15.

\section{Ready to submit your research? Choose BMC and benefit from:}

- fast, convenient online submission

- thorough peer review by experienced researchers in your field

- rapid publication on acceptance

- support for research data, including large and complex data types

- gold Open Access which fosters wider collaboration and increased citations

- maximum visibility for your research: over $100 \mathrm{M}$ website views per year

At BMC, research is always in progress.

Learn more biomedcentral.com/submissions 